\title{
Cyclic Period Changes of $\beta$ Lyrae-type Eclipsing Variable Stars KR Cyg, V382 Cyg and BX And
}

\author{
D. E. Tvardovskyi ${ }^{1 *}$ V.I. Marsakova ${ }^{2 \dagger}$ \\ ${ }^{1}$ Richeleu liceum, Elisavetinskaya, 5, Odessa, 65082, Ukraine \\ ${ }^{2}$ Astronomical Department, I. I. Mechnikov Odessa National University, Marazliyivska, 1v, Odessa, 65014, Ukraine
}

\begin{abstract}
We have studied three binary stellar systems KR Cyg, V382 Cyg and BX And, which are eclipsing variables of the $\beta$ Lyrae - type. We have studied the phase curves and $\mathrm{O}-\mathrm{C}$ curves. The forms of their O-C curves are sinusoidal with a trend, so we made the conclusion that the periods of binary systems change periodically and we suppose that the systems have third components that do not take part in the eclipses, but produce a periodic "light time" effect, as the inner eclipsing binary rotates around the common centre of masses of the triple system. For the analysis, we used data published in the AAVSO and NSVS databases, as well as our own observations. For the O-C curves, the data were taken from the BRNO database, as well as from our own calculations of the moments of minima from the light curves. We determined secondary periods, calculated the minimal masses of the third components, estimated the mass transfer rate for the system V382 Cyg and corrected the ephemeris for these three eclipsing variables.
\end{abstract}

Key words: eclipsing binaries, $\beta$ Lyr (EB) type, O-C curve, phase curve, semi-detached system

\section{INTRODUCTION}

Three variables: KR Cyg, V382 Cyg and BX And drew our attention due to significant shifts of minima of the phase curves in different cycles. The O-C curves corroborate this observation. The form of OC curves was sinusoidal with a linear trend, so we made the conclusion that the periods of these binary systems change cyclically. One of the processes that leads to such changes may be the motion of the close eclipsing binary system around the common centre of mass of the complete triple system (e.g. $[10,5])$.

\section{THE METHODS}

For our analysis, we used the visual and V-band data from the database of the "American association of variable star observers" $\left(\mathrm{AAVSO}^{1}\right)$, where original observations of many amateur observers around the world are collected. Also, for KR Cyg, we got the moment of minima by using our own observations at the Astrocamp "Variable-2015"2. For BX And the average moment of minima was obtained by using the CCD-observations from Northern Sky Variability Survey (NSVS). For calculations of the moments of minima, we used the polynomial fit realised in the MCV program [3]. Detailed description of the methods were presented in $[1,2,4]$. Results of our analysis are given in Table 1.

\footnotetext{
*dtvardovskyi@ukr.net

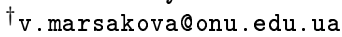

${ }^{1}$ http: //ww .aavso.org/

${ }^{2}$ http: //www .astrokolonica.sk/joomla15/en/variable.html

${ }^{3}$ http: //var.astro.cz/ocgate/

D. E. Tvardovskyi, V.I. Marsakova, 2015
}

For the O-C analysis, we used the moments of minima, which we determined from the time series, and the published ones taken from the database of the "Brno Regional Network of Observers" $\left(\mathrm{BRNO}^{3}\right)$. We also used the program MCV [3] to approximate the O-C curve and, by using the parameters of its linear trend, to calculate a more precise ephemeris (Table 2) than that given in the GCVS [6], thanks to a larger volume of analysed data.

\section{$\mathrm{KR}$ CYG}

GCVS ephemeris are given in Table 2. Using photometric data, Sipahi [7] concluded that KR Cyg is a near-contact binary system with a large effective temperature difference between the components. We have only primary minima of $\mathrm{KR} \mathrm{Cyg} \mathrm{at} \mathrm{the} \mathrm{phase}$ curve (see Fig. 1). This phase curve was built using our corrected ephemeris (Table 2 ).

We obtain the $\mathrm{O}-\mathrm{C}$ curve using our moments of minima and the ones collected from the BRNO database, as described in the previous paragraph. The O-C curve has a sinusoidal form with a superimposed inclined line (trend). Using the periodogram analysis with taking into account the linear trend, we estimated a period of about 30000 days (about 80 years, see Table 3 ). Other authors [7] also found the "quasi-periodic variation" of the O-C curve with a period of about 76 years (about 27000 days) and 
explained them as an impact of the third body in the system.

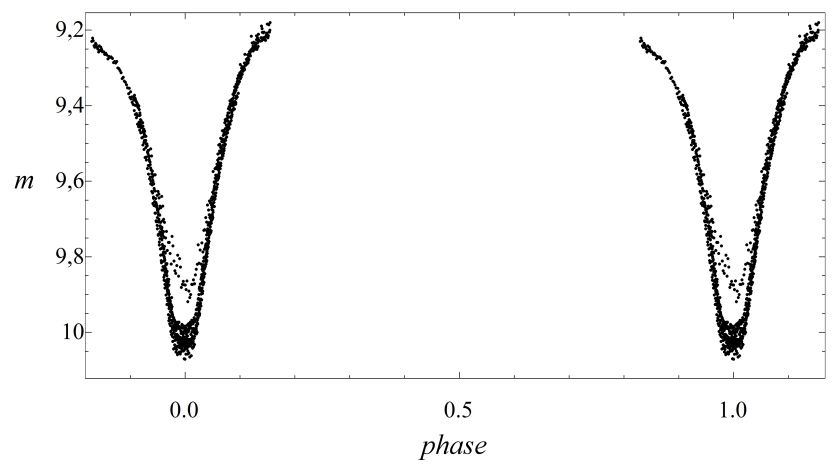

Fig. 1: Phase curve of KR Cyg built using the CCDobservations in V-band from the AAVSO database.

The parameters of the close binary system were taken from [8] and were used for calculation of the mass of the third component. According to this article, in which the analysis of the radial velocity curves was made, the masses of the primary and secondary components are 2.88 and $1.26 \mathfrak{M}_{\odot}$, respectively.

The parameters of sinusoidal components in O-C changes are given in Table 3 .

\section{V382 CYG}

The $\mathrm{O}-\mathrm{C}$ curve is shown at Fig. 2. The form of the O-C curve represents a parabola with the imposition of the sinusoid, more complicated than the model "line+sinusoid", but also available in the MCV code [3].

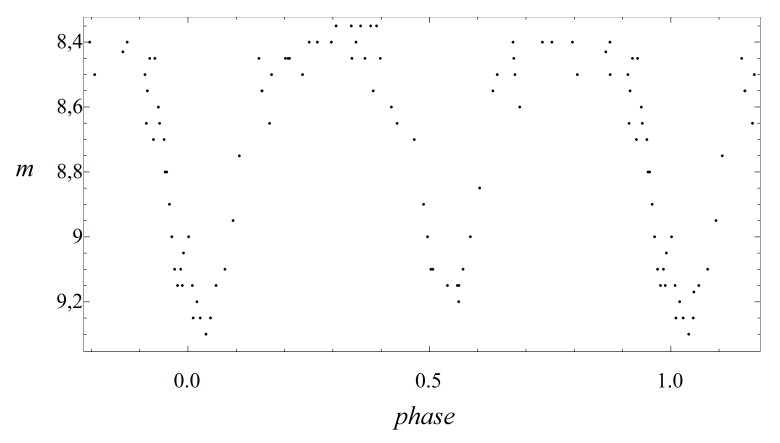

Fig. 2: Phase curve of V382 Cyg built by using the visual observations of Adam Derdzikowski, one of the AAVSO observers.

Due to the parabolic form of the curve, we made the conclusion that the increase of the period can be explained by the mass transfer from the less massive component to the more massive one [12]. We used the formula (e.g. [10]):

$$
\dot{M}=\frac{M_{1} M_{2}}{3\left(M_{1}+M_{2}\right)} \frac{\dot{P}}{P}
$$

to calculate the rate of mass transfer $\dot{M}$. Here $\dot{P}$ is the rate of period increase, and $M_{1}, M_{2}$ are masses of stars in the close binary system.

After performing the calculations, we obtained the result: $\dot{M}=6.1 \cdot 10^{-6} \mathfrak{M}_{\odot} /$ year, which is in good agreement with the value of [12]. From this paper we took the masses of the components (27.9 and $\left.20.8 \mathfrak{M}_{\odot}\right)$ and the orbital characteristics for our further calculations. We determined the period of the sinusoidal component of about 18700 days (see Table 3).

\section{BX AND}

BX And also is known as a near-contact binary system with a large effective temperature difference between components and with masses of primary and secondary components 2.15 and $0.98 \mathfrak{M}_{\odot}$, respectively [9]. The form of the O-C curve is also sinusoidal with a linear trend. We determined the period of approximately 27500 days (see Table 3 ).

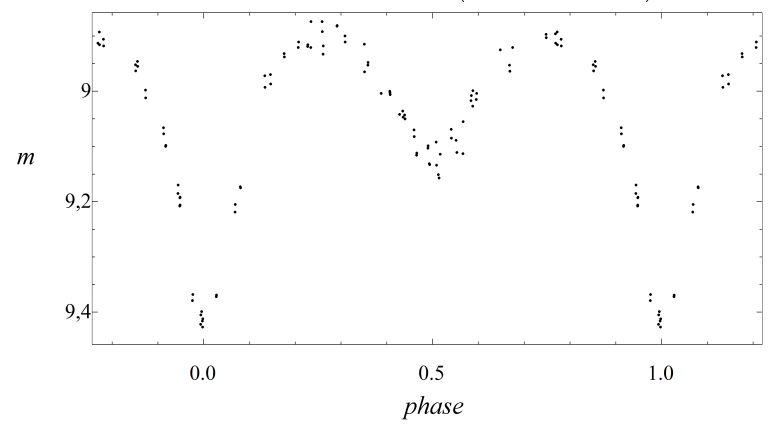

Fig. 3: Phase curve of BX And built by using the R-band observations of NSVS [11].

\section{THE THIRD COMPONENT MASSES}

We also calculated the minimal mass of the third component in each system using the "light-time" effect. We consider the system where two components of eclipsing binary are close to each other and the third one has a much wider circular orbit (e.g.[10]). Its major semi-axis can be derived using the third Kepler's law:

$$
A^{3}=\frac{G M T^{2}}{4 \pi^{2}}=\frac{G\left(M_{1}+M_{2}+M_{3}\right)}{\omega^{2}}
$$

where $A$ is the major semi-axis of the third component relatively to the binary system, $G$ is the gravitational constant; $T$ is the orbital period of the third body (and the period of O-C changes), $\omega=2 \pi / T$ is the angular speed, $M=M_{1}+M_{2}+M_{3}$, where $M_{1}$, $M_{2}, M_{3}$ are the masses of the components.

Law of the barycenter motion gives us the formula for the projection on the line of sight of the major semi-axis of binary system orbit relatively to 
the barycenter $a_{12}$ :

$$
a_{12}=c \Delta t=A \frac{M_{3}}{\left(M_{1}+M_{2}+M_{3}\right)} \sin i,
$$

where $c$ is the light speed, $\Delta t$ is the time delay of light propagation on the way to us and it corresponds to the semi-amplitude of the $\mathrm{O}-\mathrm{C}$ curve, $i$ is the angle of inclination of the third component's orbit (angle between orbit of the third component and the plane of the sky that is perpendicular to the line of sight).

Then we can obtain:

$$
\sin i=\frac{c \Delta t\left(M_{1}+M_{2}+M_{3}\right)}{A M_{3}} .
$$

Then we tabulated mass of the third component $\left(M_{3}\right)$ with a small step and calculated the angle of inclination of its orbit by these formulas $(3,4$ and 5). For tabulating, we have used the Excel tables. Since the value of the sine of the angle cannot be more than 1 , this gives us the limit of the minimal mass of the third component.

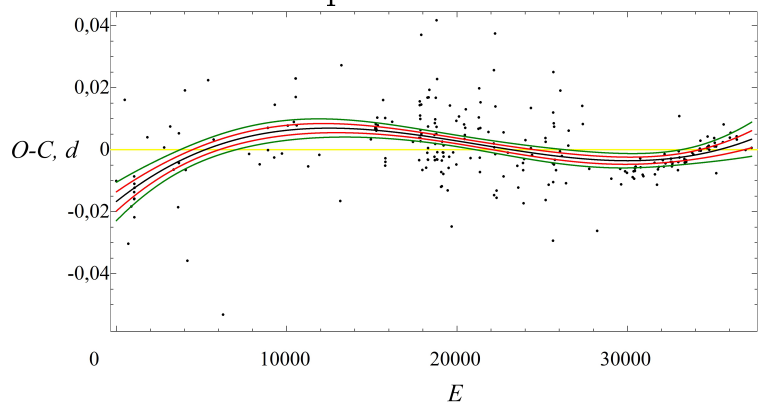

Fig. 4: O-C curve for KR Cyg.

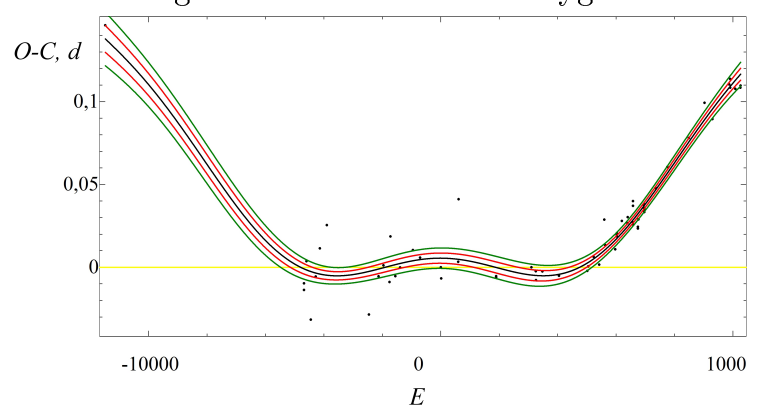

Fig. 5: O-C curve for V382 Cyg.

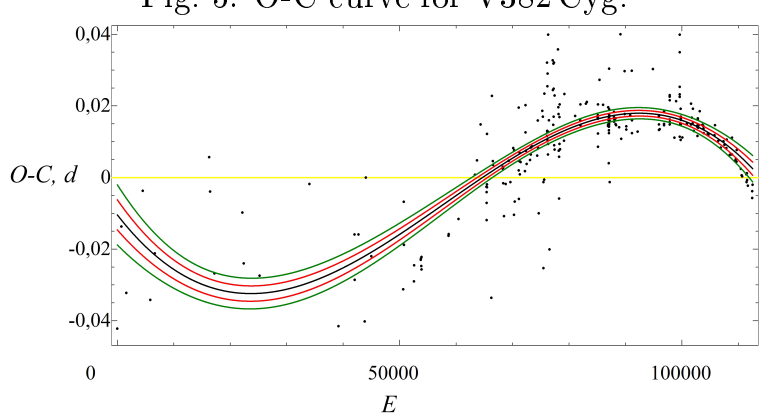

Fig. 6: O-C curve for BX And.

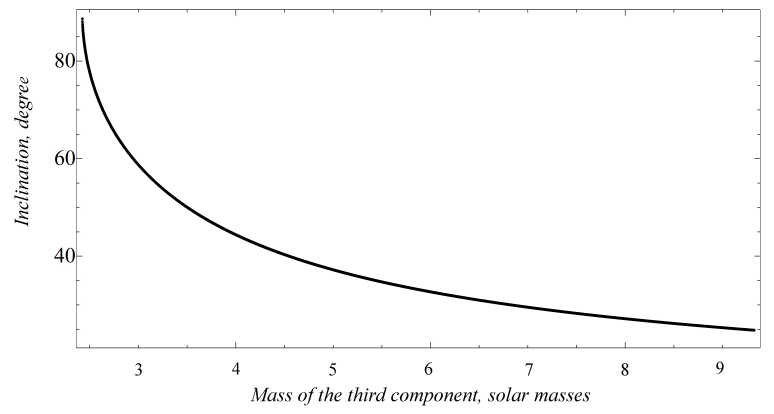

Fig. 7: Example of relation between inclination $(i)$ and the third component mass $M_{3}$ for KR Cyg.

\section{RESULTS AND CONCLUSIONS}

We have studied three eclipsing-binary stars: KR Cyg, V382 Cyg and BX And. For these three systems, we made the conclusion on the periodical changes of the O-C (see Table 3) and suggested the presence of the third component in each of these three systems. The $(O-C)$ values were approximated by a function

$$
\begin{aligned}
O-C=\left(\sum_{j=0}^{s} C_{j} \cdot\right. & \left.\left(E-E_{0}\right)^{j}\right)+ \\
& +R \cdot \cos \left(2 \pi\left(E-E_{\max }\right) / P_{2}\right),
\end{aligned}
$$

where $R$ is (semi)amplitude of the long-period variations, $P_{2}$ is a long (secondary) period, and the "initial epoch" $E_{\max }$ corresponds to the maximum of the cosine function. It may be converted to Julian dates as $T_{\max }=T_{0}+P_{0} \cdot E_{\max }$, where $T_{0}$ is given in the second column of Table 2 .

For all three systems, we have calculated the minimal mass of the third component and we got the following results:

- In the KR Cyg system: $M_{3} \geq 2.411 \mathfrak{M}_{\odot}$;

- In the V382 Cyg system: $M_{3} \geq 2.582 \mathfrak{M}_{\odot}$;

- In the BX And system: $M_{3} \geq 0.513 \mathfrak{M}_{\odot}$.

We also computed the diagrams which demonstrate the relation between mass of the third component and the inclination angle for all systems studied. The example of such diagram is shown in Fig. 7.

We also corrected the ephemerides taking into account the linear or parabolic trend of the O-C curves. Results are presented in Table 2.

As alternative hypotheses of the reasons leading to the periodic changes of the $\mathrm{O}-\mathrm{C}$, one may suggest e.g. the alternating transfer of masses (which seems physically not reasonable for the $\beta$ Lyr-type stars, as in them only one component fills its Roche lobe, e.g. [10]) as well as the pulsations of one of the components in the binary system (but there is no evidence for such high-amplitude oscillations at the light curves). 
Table 1: Calculated moments of minima.

\begin{tabular}{cccccc}
\hline system & Cycle number & JD & \pm & degree of polynomial & band (and the source of data) \\
\hline KR Cyg & 34690 & 2455018.775 & 0.002 & 9 & V(AAVSO) \\
KR Cyg & 34814 & 2455123.577 & 0.002 & 9 & V(AAVSO) \\
KR Cyg & 35117 & 2455379.659 & 0.002 & 9 & V(AAVSO) \\
KR Cyg & 35214 & 2455461.6356 & 0.0003 & 9 & V(AAVSO) \\
KR Cyg & 35660 & 2455838.5809 & 0.0009 & 9 & V(AAVSO) \\
KR Cyg & 36066 & 2456181.715 & 0.001 & 9 & V (AAVSO) \\
KR Cyg & 36417 & 2456487.6565 & 0.0006 & 9 & R(AAVSO) \\
KR Cyg & 33050 & 2453300.5727 & 0.0008 & 9 & R(Variable) \\
KR Cyg & 37295 & 2457220.399 & 0.001 & 9 & Vis(AAVSO) \\
\hline V0382 Cyg & 9905 & 2455490.972 & 0.001 & 9 & R(NSVS) \\
\hline BX And & -1547 & 2451540.0293 & 0.0008 & 9 & \\
\hline
\end{tabular}

Table 2: The GCVS ephemerides and the corrected ones (without cosine term).

\begin{tabular}{ccc}
\hline Variable & GCVS ephemeris [6] & New ephemeris \\
\hline KR Cyg & $T=2429106.413+0.8451517 \cdot E$ & $T=2425700.4320 \pm 0.0006+(0.84515267 \pm 0.00000007) \cdot E$ \\
V382 Cyg & $T=2436814.7706+1.8855146 \cdot E$ & $T=2436814.7581 \pm 0.0016+(1.8855147 \pm 0.0000002) \cdot E+$ \\
& & $+(1.10 \pm 0.04) \cdot 10^{-9} \cdot E^{2}$ \\
BX And & $T=2452500.3454+0.6101119 \cdot E$ & $T=(52500.3462+-0.0007)+(0.61011357 \pm 0.00000005) \cdot$ \\
\hline
\end{tabular}

Table 3: Characteristics of the sinusoidal approximation of $\mathrm{O}-\mathrm{C}$ variations.

\begin{tabular}{ccccc}
\hline Variable & Initial epoch, $E_{0}$ & Period of O-C changes, $P_{2}$ & Semi-amplitude, $R$ \\
\hline KR Cyg & cycles & cycles & days & days \\
\hline V382 Cyg & $-79 \pm 7 \pm 2460$ & $35497 \pm 8690$ & $30000 \pm 7344$ & $0.0074 \pm 0.0029$ \\
BX And & $-10492 \pm 510$ & $43933 \pm 1627$ & $27540 \pm 1087$ & $0.0220 \pm 0.0017$ \\
\hline
\end{tabular}

\section{ACKNOWLEDGEMENT}

We sincerely thank all members of AAVSO and BRNO communities for their work that made this researches possible. We also grateful to the NSVS project for their observations. The thank Ivan L. Andronov for helpful discussions. We express special thanks to the organizers of the Astrocamp "Variable2015" and especially to Pavol A. Dubovsky for helpful training in obtaining CCD-observations.

\section{REFERENCES}

[1] Andronov I. L. 2003, ASP Conf. Ser., 292, 391

[2] Andronov I. L. 2005, ASP Conf. Ser., 335, 37

[3] Andronov I. L. \& Baklanov A. V. 2004, Astronomy School Rep., 5, $264^{4}$
[4] Andronov I. L. \& Marsakova V. I. 2006, Astrophysics, 49, 370

[5] Rucinski S. 2010, AIP Conf. Proc., 1314, 29

[6] Samus N. N., Durlevich O. V., Kazarovets E. V. et al. 2012, General Catalog of Variable Stars (GCVS database, Version 2012Jan $)^{5}$

[7] Sipahi E. 2012, New Astronomy, 17, 383

[8] Sipahi E., Çakirli Ö. \& Ibanoglu C. 2013, RMxAA, 49, 25

[9] Siwak M., Zola S. \& Koziel-Wierzbowska D. 2010, Acta Astronomica, 60, 305

[10] Tsessevich V.P. (ed.) 1971, 'Eclipsing Variable Stars', Moscow, Nauka

[11] Woźniak P. R., Vestrand W. T., Akerlof C. W. et al. 2004, AJ, 127, 2436

[12] Yaşarsoy B. \& Yakut K. 2013, AJ, 145, 9

\footnotetext{
${ }^{4}$ http://uavso.pochta.ru/mcv

${ }^{5}$ http://www.sai.msu.su/gcvs/gcvs/
} 